\title{
Intraoperative ultrasonographic characteristics of malignant intracranial lesions
}

\author{
Çokluk Cengiz, Aydin Keramettin \\ Department of Neurosurgery, Medical Faculty, Ondokuzmayis University, Samsun, Turkey
}

\begin{abstract}
Aims: The aim of this study was to evaluate the capability of intraoperative ultrasonography (IOUSG) in identifying malignant intraparenchymal tumors during surgical intervention. Settings and Design: Forty patients with intrinsic malignant tumors were evaluated by using IOUSG. Materials and Methods: A real-time ultrasound scanner with a 3- or 5-MHz transducer was used for this study in all cases. The tip of the ultrasound probe was placed on the intact cranial dura mater and then moved in the sagittal and coronal planes. Results: Nineteen of forty patients had lesions that were primary malignant glial tumors. Five of them had previous surgery and radiotherapy. The remaining twenty-one had metastatic tumors. All lesions were well localized and malignant characteristics were well defined by IOUSG. Radiationinduced changes in five lesions in the surrounding brain and tumor parenchyma were described. Conclusion: In conclusion, IOUSG is not only helpful in localizing lesions but it can also be used in determining the malignant characteristics of lesions.
\end{abstract}

Key Words: Intracranial tumors, intraoperative ultrasonography, malignant brain lesions

Ultrasonography has been used as an intraoperative diagnostic tool since $1970 .{ }^{[1]-[10]}$ In this study, we investigated and described the ultrasonographic characteristics of malignant lesions, and evaluated the capability of sonography for this purpose.

\section{Materials and Methods}

This retrospective clinical study was approved by the ethical committee of the Ondokuzmayis University, (Medical Faculty).

The study population comprised patients with primary malignant glial and metastatic tumors. Inclusion criteria were as follows in the preoperative stage: (1) previously operated and irradiated recurrent malignant tumors, (2) metastatic malignant lesions originated from another part of the body, (3) intaparenchymal location on computer- ized tomography (CT) and magnetic resonance imaging (MRI), (4) perilesional edema appearing on CT and MRI, and (5) cystic and/or necrotic parts demonstrated by CT and MRI. At the intraoperative stage, fast histopathological examination (Frozen section) was performed for all lesions. Benign intracranial lesions were excluded from this study.

Forty patients (between 25 and 75 years of age, mean age \pm SD; $53.4 \pm 12$ ), who underwent surgery for malignant brain tumors during the period between August 2000 and July 2001, were investigated. Twenty-five (62\%) patients were male and 15 (38\%) female.

Computerized tomography and MRI were performed in all cases at the preoperative stage.

All tumors were evaluated by one of the authors (CC) using a Tosbee real-time ultrasound scanner (Tosbee, Toshiba Inc., Tokyo). A 3- or $5-\mathrm{MHz}$ transducer was used. The transducer and its cord were covered with a sterile, translucent, disposable plastic sheath to maintain sterile surgical conditions. Before covering the system, the tip of the transducer head was plastered with sterile gel to maximize sonographic images. The tip of the transducer was placed gently on the intact cranial dura and moved in the direction of the sagittal and coronal planes. The selected images were printed.

The tumor location under the craniotomy, the margins from the perilesional brain tissue, internal configuration and echogenicity, the changes in the peritumoral area, and the location of the neuroanatomical structures such as the ventricle, falx, and main arteries to the border of the lesions were also imaged and printed.

After gross resection of tumors, the ultrasonographic examination was repeated to check tumor bed and the residual tumor.

Descriptive statistical analysis was used in this study.

\section{Results}

Of the 40 lesions analyzed in this study, 19 (47\%) were intrinsic primary malignant glial tumors and $21(53 \%)$ were metastatic tumors. Computerized tomography and MRI showed irregular contour, irregular contrast enhancement, necrotic parts, and perilesional edema in all patients. The distribution of lesions is shown in Table 1.

Thirty-two cases showed a cystic part on CT and MRI. The ultrasonographic appearance of malignant cysts was as low echogenic areas. Free necrotic particles and double density 
were commonly present in malignant cysts. The solid component surrounding the cysts was thick. The external surface of solid parts was regular but internal surfaces facing cysts were irregular, sometimes including the part projecting into the cyst. Figure 1 demonstrates general features of malignant cysts.

Sixty-three percent of the patients had multiple necrotic parts, and the remaining $37 \%$ had a single necrosis located in the central part of the lesion. The necrotic part of malignant lesions can be solitary or multiple, and has a mixechogenic appearance. The most hypoechogenic part was commonly located in the central portion. The shape and configuration of the necrotic part may be different from one to another type of malignant lesion. Some characters of necrotic tumors are seen in Figure 2.

All malignant lesions showed perilesional edema on CT and MRI. In intraoperative sonographic examination, malignant perilesional areas were seen as two different echogenic zones. The tumors were surrounded by a first, very thin hypoechogenic zone. The echogenicity of the first zone was similar to brain tissue. The second zone was a vasogenic edematous zone. The ultrasonographic appearance of this zone was hyperechogenic and was similar to that of solid tumor parts. The ultrasonographic appearance of perilesional areas, and solid and necrotic parts is shown in Figure 3.

Neuroradiological examination showed that perilesional edema, necrosis, irregular contour, and contrast enhancement were present in all previously irradiated recurrent intrinsic glial tumors.

The ultrasonographic appearance of five previously irradi-

\begin{tabular}{ll}
\hline \multicolumn{2}{c}{ Table 1: The distribution of the lesions } \\
\hline Malignant lesions & Number \\
High-grade gliomas & $19(47)$ \\
Previously operated on and irradiated & $3(15)$ \\
First operation & $16(75)$ \\
Metastatic tumors & $21(53)$ \\
Lung & $11(52)$ \\
Breast & $5(24)$ \\
Gastrointestinal tract & $5(24)$
\end{tabular}

Values in parenthesis are in percentage.

\begin{tabular}{|c|c|}
\hline \multicolumn{2}{|c|}{$\begin{array}{l}\text { Table 2: The main characteristics of malignant lesions } \\
\text { detected by intraoperative ultrasonography (IOUSG) }\end{array}$} \\
\hline Main component & Malignant characteristics \\
\hline \multirow{5}{*}{ Necrotic part } & Single or multiple necrosis \\
\hline & Surrounded by solid part of tumor \\
\hline & Contains tumor particles \\
\hline & Irregular internal wall \\
\hline & Located central or paracentral \\
\hline \multirow[t]{2}{*}{ Cystic part } & Single or trabeculated \\
\hline & $\begin{array}{l}\text { Surrounded by thick solid component } \\
\text { Echogenicity is very low }\end{array}$ \\
\hline \multirow[t]{2}{*}{ Tumor contour } & Irregular contour \\
\hline & Invasive growth pattern \\
\hline \multirow[t]{3}{*}{ Perilesional changes } & $\begin{array}{l}\text { Vasogenic edema is very common and } \\
\text { dense }\end{array}$ \\
\hline & Tumor infiltration is present \\
\hline & Poor demarcation line \\
\hline
\end{tabular}

ated malignant glial tumors was hyperechogenic compared to the surrounding brain. Our observations showed that the density of peritumoral edemas was lower than those of unradiated cases and that the density of the brain is lower than that of normal brain. The growth pattern of tumors was demonstrated to be of both distortive and invasive character. Figure 4 demonstrates some characteristic features of radiation-induced changes in the brain and tumor.

The main ultrasonographic characteristics of malignant brain lesions are shown in Table 2.

\section{Discussion}

This study indicates that intraoperative ultrasonographic examination is not only useful in the location of cystic, necrotic and solid parts of tumors but may also distinguish malignant features of lesions at the beginning of surgery. Preoperative CT and MRI may reveal information about the pathological nature of the lesion. The features obtained from these neuroradiological modalities, such as necrotic parts, perilesional edema, irregular contour, and irregular contrast enhancement can be considered as the malignant characteristics [Table 3]. Previous articles have reported that sonography can be used in the location of tumors, definition of their margins, differentiation of internal characteristics, and detection of residual tumors. ${ }^{[3]-[12]}$ Intraoperative MRI is clearly superior to intraoperative ultrasound examination to investigate the completeness of tumor resection during surgery, but it is much cheaper and thus more easily available. It is also shown that IOUSG can enhance the orientation of the surgeon by showing the depth, size, location, and characteristics of the lesion. ${ }^{[6],[7]}$ Intraoperative ultrasonography may also be used as intraoperative image guidance in figuring out 'where am I' after splitting of the brain parenchyma and dissection of the brain sulcus. We usually use small cotton paddy during this stage. The major constraint of ultrasonographic examination

\begin{tabular}{lc}
\hline \multicolumn{2}{c}{ Table 3: The details of the patients } \\
\hline Number of the patients & 40 \\
Mean age \pm SD & $53.4 \pm 12$ \\
Sex & $25(62)$ \\
Male & $15(38)$ \\
Female & \\
Pathology & $19(47)$ \\
High-grade gliomas & $21(53)$ \\
Metastatic tumors & \\
Preoperative computerized tomography and & magnetic resonance \\
imaging & $32(80)$ \\
Cystic & $40(100)$ \\
Necrotic & $40(100)$ \\
Perilesional edema & \\
Intraoperative ultrasonographic findings & $32(80)$ \\
Cystic & $40(100)$ \\
Necrotic & $40(100)$ \\
Perilesional edema & $40(100)$ \\
Irregularly tumor contour &
\end{tabular}

Values in parenthesis are in percentage. 

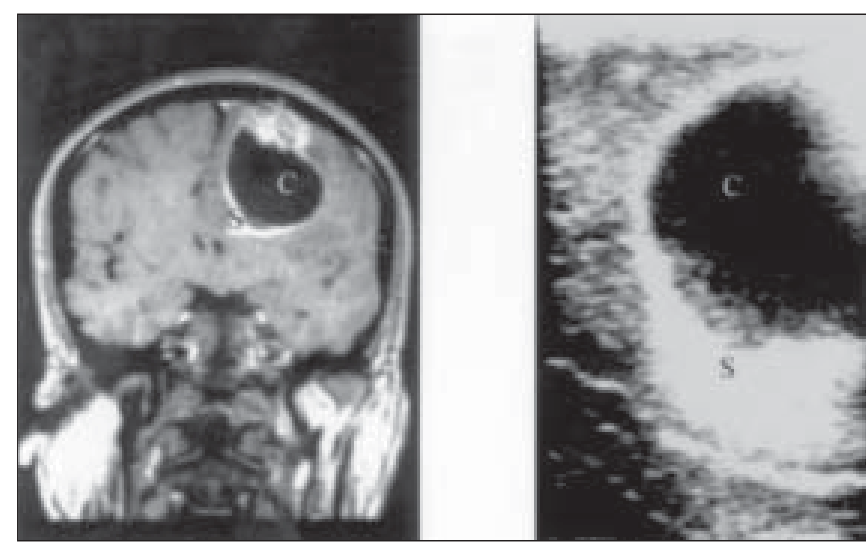

Figure 1: A 57-year-old-male with high-grade glial tumor (anaplastic astrocytoma). (A) T1-weighed and Gd-enhanced magnetic resonance imaging (MRI) shows the cystic tumor located in the left parietal lobe. C, cyst; S, solid part of the tumor. (B) The solid and cystic parts of the tumor can easily be distinguished by intraoperative ultrasonography (IOUSG). Internal and external irregular contours, a thick, solid part, and invasive growth pattern along the brain tissue suggest a malignant tumor. C, cyst; S, solid part of the tumor
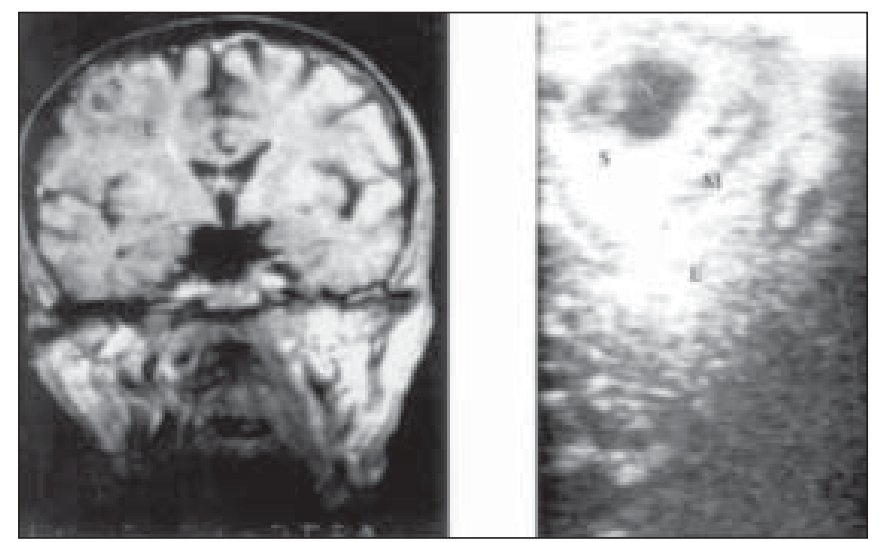

Figure 3: This is an example of metastatic lesion of a malignant lung tumor. (A) T1-weighed, Gd-enhanced magnetic resonance imaging (MRI) shows the right parietal mass lesion with perilesional edema. S, solid part; E, edema. (B) Intraoperative ultrasonography (IOUSG) shows a single necrosis surrounded by the solid part of the tumor, including tumor particles, irregular tumor margin, invasive growth pattern, dense vasogenic edema, and poor demarcation line. $\mathbf{N}$, necrotic part; S, solid part; $M$, margin of the tumor; $E$, vasogenic edema

is that it requires a high level of skill and experience in the investigator. Surgical removal is especially difficult in lowgrade glial tumors due to poor demarcation between lesion and brain. ${ }^{[13]}$ Intraoperative ultrasonography can provide diagnosis of the demarcation line, border of lesion, and edematous tissue. ${ }^{[6],[7],[13]}$ The borders of malignant tumors have features different from those of benign tumors and they can be easily detected by IOUSG.

The capsule is the best demarcation line for intrinsic and extrinsic lesions. However, this is not always the case as some noncapsular tumors can be well demarcated from surrounding neural tissues. The external surface of the capsule is the best area for surgical retraction and dissection. The echogenicity of the capsule is usually denser than that of the solid part of the tumor. However, sometimes it is difficult to distinguish between these two parts. The configuration of the

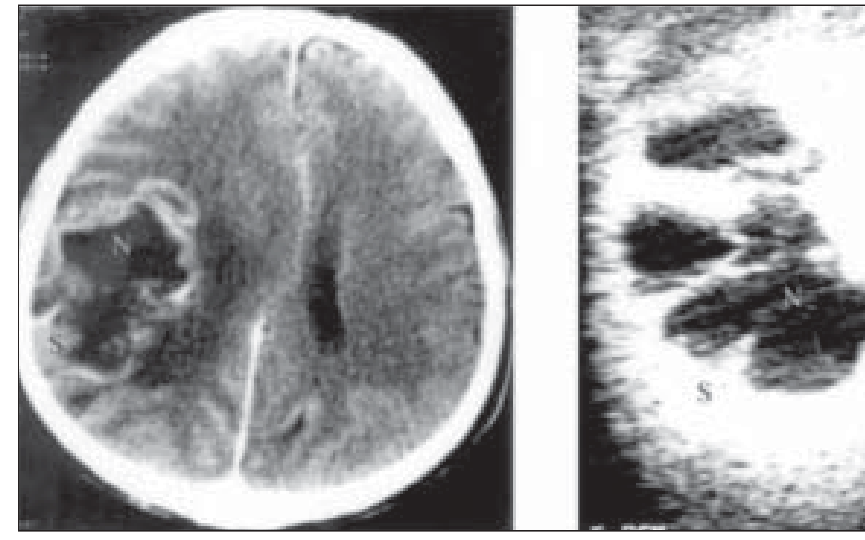

Figure 2: A 59-year-old-male with high-grade glial tumor (glioblastome multiforme). (A) Computerized tomography (CT) shows a necrotic tumor located in the right parietal region. N, necrotic part; S, solid part (B) Intraoperative ultrasonography shows compartmentalization of the necrotic part and solid area of the tumor. N, necrotic part; S, solid part
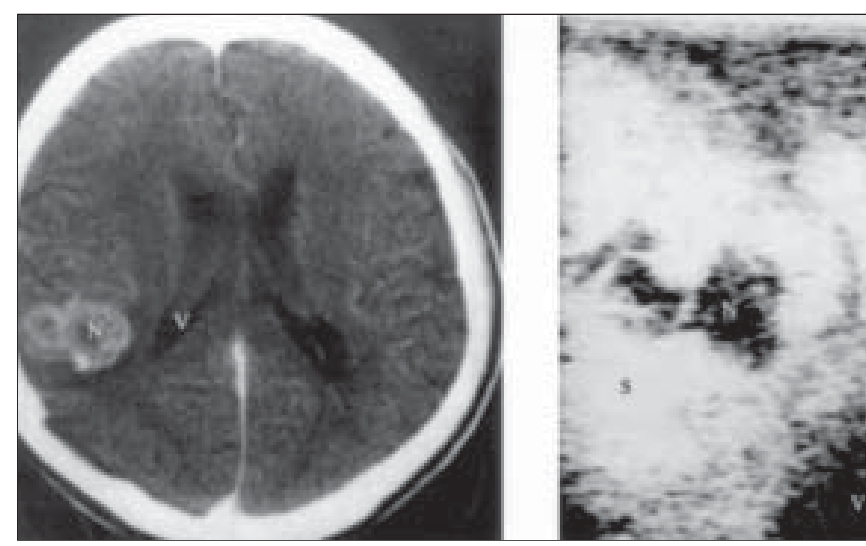

Figure 4: A 57-year-old-male with a previously operated on high-grade glial tumor (anaplastic astrocytoma). After surgery, the patient received radiotherapy. (A) Computerized tomography (CT) shows a recurrent tumor in the left parietal region. $\mathrm{N}$, necrosis; S, solid part; V, ventricle.

(B) Intraoperative ultrasonography shows the necrotic part with irregular internal wall, irregular tumor contours, and hypoechogenic brain tissue without vasogenic edema. $\mathrm{N}$, necrosis; S, solid part; $\mathrm{V}$, ventricle

capsule is usually irregular in malignant tumors but tends to be more regular and smooth in benign tumors.

High-grade glial tumors generally have irregular margins. This feature reflects their invasive character. Surgical dissection of these tissues from the brain is extremely difficult. This is similar with those of some metastatic tumors. However, many metastatic lesions have regularly configured borders. Some malignant tumors may have both regular and irregular parts throughout their margin.

Necrotic components are dead parts of lesions and commonly found in malignant tumors. They do not have any vital metabolic reactions and blood circulation. The sonographic appearance of these parts is as low echogenic areas located somewhere in the tumor parenchyma. The necrotic parts are always surrounded by a solid part. Their margins are irregular. Dead necrotic pools may have some tumor particles in their 
fluid. During sonographic examination, these particles can be seen as freely moving particles when using the sonographic transducer with light pressure. The necrotic part of tumors is generally located in the central or paracentral area. But this concept is not true in tumors that generally originate and are fed from one area. In this type of tumor, the necrotic parts are located in the most remote part of the feeding point.

Other dead parts of tumors are cysts. The characteristic sonographic appearance of cysts is as hypoechogenic areas. Cysts can be associated with benign and malignant tumors. Sonographically, cysts are seen as hypoechogenic areas totally enclosed by a solid part or a membranous structure. Malignant tumors usually have thick a wall, and are commonly associated with malignant perilesional changes. Our ultrasonographic experience of benign cystic lesions is that they have very thin, hyperechogenic cystic walls without perilesional changes. Cysts do not have any gross particles in their fluid. The inner surface of the cyst wall is generally smooth and sometimes associated with trabecules.

Malignant perilesional areas have two different echogenic zones. The first ribbon-like zone encloses the mass and does not include vasogenic edema. The ultrasonographic appearance is hypoechogenic compared to normal brain, but hyperechogenic compared to those of CSF. The zone is compressed by the mass effect of the lesion. The elasticity and biomechanical properties are changed by the growing mass of tumors. ${ }^{[14],[15]}$ Ischemic and gliotic changes are present in this zone. ${ }^{[14]-[16]}$ Its blood supply is decreased by the tumor mass. ${ }^{[17]}$ The second zone is a vasogenic edema zone that begins at the border of the first zone. The echogenicity of this zone is similar to solid parts of tumors. The edema associated with metastatic and primary brain tumors is an extracellular vasogenic edema, which extends along the white matter. ${ }^{[14]-[18]}$ The cause of this edema is disruption or defective function of the bloodbrain barrier. It is usually steroid responsive. There is no relationship between tumor size and the density of the edema. Even small tumors can be associated with aggressive and dense hyperechogenic edema. The density of the edema gradually decreases with increasing distance from the tumor. Malignant perilesional edema is irregular, has a finger-like shape, and can easily be detected by ultrasonography.

\section{Conclusions}

Intraoperative ultrasonography can easily detect the location of intracranial tumors in the brain. We investigated the criteria for distinguishing malignant lesions. Important information about the characteristics of brain tumors can be obtained by ultrasonographic examination of the lesions. The contour of tumors and perilesional changes are the main characteristics. The presence of cysts and necrosis and their characteristics are also important.

Irregular border, necrotic parts, invasive growth pattern, and dense perilesional edema can all be attributed to malignancy.

\section{References}

1. Lang FF, Sawaya R. Surgical management of cerebral metastases. Neurosurg Clin of North Am. 1996;7:459-84

2. Ya ${ }^{\circ}$ argil MG. Microneurosurgery. CNS Tumors: Surgical anatomy, neuropathology, neuroradiology, neurophysiology, clinical considerations, operability, treatment options. $2^{\text {nd }}$ Ed. New York: Georg Thieme verlag; 1994.

3. Auer LM, van Velthoven V. Intraoperative ultrasound (US) imaging. Comparison of pathomorphological findings in US and CT. Acta Neurochir (Wien) 1990;104:84-95.

4. Brownbill D. The clinical value of echoencephalography. Aust NZ J Surg $1970 ; 40: 74-8$

5. Glasauer FE, Schlagenhauff RE. The use of intraoperative echoencephalography. Neurology 1970;20:1103-7.

6. Gronningsaeter A, Unsgard G, Ommedal S, Angelsen BA. Ultrasound-guided neurosurgery: A feasibility study in the $3-30 \mathrm{MHz}$ frequency range. $\mathrm{Br} \mathrm{J}$ Neurosurg 1996;10:161-8.

7. Hammoud MA, Ligon BL, elSouki R, Shi WM, Schomer DF, Sawaya R. Use of intraoperative ultrasound for localizing tumors and determining the extent of resection: A comparative study with magnetic resonance imaging. J Neurosurg. 1996;84:737-41.

8. Kumar P, Sukthankar R, Damany BJ, Mishra J, Jha AN. Evaluation of intraoperative ultrasound in neurosurgery. Ann Acad Med Singapore $1993 ; 23: 422-7$.

9. Szczerbicki M, Jarek K, Kozlowski J. Intraoperative ultrasonography of brain tumors. Neurol Neurochir Pol 1996;30:831-8.

10. Tada M, Kaneko S, Imai T, Abe H, Tsuru M, Shirato H, et al. Intraoperative sonography for brain tumor surgery. Hokkaido Igaku Zasshi 1985;60:82-90.

11. Knake JE, Bowerman RA, Silver TM, McCracken S. Neurosurgical applications of intraoperative ultrasound. Radiol Clin North Am 1985;23:73-90.

12. Quencer RM, Montalvo BM. Intraoperative cranial sonography. Neuroradiology $1986 ; 28: 528-50$.

13. Knake JE, Chandler WF, Gabrielsen TO, Latack JT, Gabarski SS. Intraoperative sonographic delineation of low grade brain neoplasms defined poorly by computed tomography. Radiology 1984;151:735-9.

14. Bertossi M, Virgintino D, Maiorano E, Occhiogrosso M, Roncali L. Ultrastructural and morphometric investigation of human brain capillaries in normal and peritumoral tissues. Ultrastruct Pathol 1997;21:41-9.

15. Kida S, Ellison DW, Steart PV, Weller RO. Characterization of perivascular cells in astrocytic tumours and peritumoral oedematous brain. Neuropathol Appl Neurobiol 1995;21:121-9.

16. Groger U, Huber P, Reulen HJ. Formation and resolution of human peritumoral brain edema. Acta Neurochir Suppl (Wien) 1994;60:373-4.

17. Strugar J, Rothbart D, Harrington W, Criscuolo GR. Vascular permeability factor in brain metastases: Correlation with vasogenic brain edema and tumor angiogenesis. J Neurosurg 1994;81:560-6.

18. Chang CC, Shinonaga M, Kuwabara T. Effect of dexamethasone on neurotransmitter amines in a rat glioma model. Adv Neurol 1990;52:483-9.

Accepted on 17-01-2005 


\section{Invited Comments}

The authors have attempted to utilize intraoperative ultrasonography to characterize criteria for malignancy of the lesions operated upon for this investigation. Of course, it was a preliminary diagnosis made on the basis of case history and preoperative imaging studies. These hypotheses, however, can be confirmed or rejected with additional information obtained by intraoperative imaging. Malignant gliomas and brain metastases were assessed. From these observations, the authors establish several criteria suggestive of malignancy during intraoperative ultrasonography. This should be useful, e.g. in an environment where frozen sections are not available. The major drawback is that nonmalignant tumours were not assessed in this study and thus, no control group is available. Ultrasonography was furthermore used to check the extent of resection. Whatever to date is the gold standard of intraoperative imaging in intrinsic brain tumours; any tech-

\section{Invited Comments}

For the past two decades, intraoperative ultrasonography (IOUS) was a well-established true real-time imaging instrument in brain surgery. Long before the advent of modern intraoperative CT and MR devices, this helpful tool could assess intraoperative orientation as well as facilitate effective tumor resection. Hence, its daily use depends on the specific experience of the user. Usually neurosurgeons are not familiar with ultrasonographic imaging to use it in their daily routine. Maybe because of this in the Scientific Echo statements heralding the benefits of the use of IOUS were low. Furthermore, the upcoming neuronavigation methods made the IOUS fall into desuetude.

Currently, modern neurosurgery is debating the benefits of neuronavigation in brain tumor surgery as the facilitation through resection via intraoperative $\mathrm{CT}$ and MR imaging. The most interesting feature for me is that modern ultrasonographic devices can offer both in one with far lower costs. There are promising data in the literature about the effectiveness of the new methods, showing that ultrasonographic resolution is equal to that of $\mathrm{CT}$ and $\mathrm{MR}$ imaging. For all the centers in the world with lesser resources, IOUS can indeed be an alternative to the expensive MR devices that have come into use.

From this angle, the present article deserves interest. Neurosurgeons have to familiarize themselves (again) with the use of intraoperative ultrasonographic devices and the imaging data they obtain before, during and after their resection work. nique will help to more precisely document the extent of surgical resection than what the surgeon feels. Technology adds information to the surgeon's estimate of the resection volume. Several techniques are available for intraoperative imaging. While ultrasonography, which is widely available, requires specific manual skill and expertise of the investigator, other intraoperative imaging techniques, such as computerized tomography or magnetic resonance scanning, are expensive and require additional space. Thus, only a minority of neurosurgeons have access to the latter technologies. However, it still remains to be shown that with more aggressive surgery, the patient's prognosis will improve.

Michael Buchfelder University of Göttingen Medical School, Göttingen, Germany. E-mail: mbuch@med.uni-goettingen.de

The intention of the authors was to characterize features of malignant brain tumors obtained from IOUS. The number of patients (40) seems to be appropriate for the purpose of comparing the sonographic appearance and configuration of cystic, solid, and necrotic parts of various malignant lesions. However, why is it important to 'predict' intraoperatively 'the pathological nature of the lesions?' What then is the value of the frozen section? And why do they not restrict their description merely to, for example, Grade IV malignant gliomas?

I am confused in the light of the fact that the study population comprised an inhomogeneous mix of patients with "previously operated and irritated recurrent tumors, metastatic malignant lesions' and true 'primary malignant glial tumors!' From my operative experience, the excision of well-delineated metastatic lesions (mostly capsulated) differs significantly from that of intrinsic gliomas.

Therefore, for me the demonstrated features such as necrotic parts, perilesional edema, irregular contour and contrast enhancement could be more of intraoperative help in orientation and consequent tumor removal than in differentiating between various histological entities. All the further results of the paper are somehow related to this, which is my major objection, so I abstain from discussing them.

Guenther Kleinpeter Neurochirurgische Abteilung, Donauspital, Langobardenstrasse 122, 1220 Vienna, Austria. E-mail:kleinpet@billrothhaus.at 